\title{
THE OPTIMAZATION OF MULTI RESOLUTION SEGMENTATION OF REMOTELY SENSED DATA USING GENETIC ALGHORITHM
}

\author{
F.Saba $^{\text {a }}$, M. J. Valadanzouj ${ }^{\text {b }}$, M. Mokhtarzade ${ }^{\text {c }}$ \\ ${ }^{a}$ Faculty of Geomatics Engineering, K.N. Toosi University, Tehran, Iran \\ saba_eng_1366@yahoo.com \\ ${ }^{\mathrm{b}}$ Faculty of Geomatics Engineering, K.N. Toosi University, Tehran, Iran \\ valadanzouj@kntu.ac.ir \\ ${ }^{\mathrm{c}}$ Faculty of Geomatics Engineering, K.N. Toosi University, Tehran, Iran \\ m_mokhtarzade@yahoo.com
}

Commission VI, WG VI/4

KEY WORDS: Object Oriented, Multi resolution Segmentation, Genetic, Optimization, IKONOS

\begin{abstract}
:
Object oriented analysis is widely used in interpretation of remote sensing images in comparison with pixel based approaches. A key step for achieving an acceptable classification result is meaningful image segmentation. Multi resolution segmentation is known as one of the most popular approaches in image segmentation that have been implemented in commercial software on the market, eCognition. However, this algorithm needs a set of optimum parameters which usually obtained by trial and error task. This technique not only is tedious and time consuming, also rely on the user's experience.

So In this study in order to alleviate this problem, genetic algorithm is proposed to find the optimal parameters for multi resolution segmentation by focusing on road feature.

This method is implemented on a pan-sharpened IKONOS image covering a part of Shiraz city, Iran. The results show that, with parameters found by GA, multi resolution segmentation accuracy is higher than obtained accuracy with parameters found by user. The evaluation of results confirms the importance of genetic algorithm to get optimal parameters.
\end{abstract}

\section{INTRODUCTION}

\subsection{Motivation}

For years, information extraction has been the topic of many researchers all around the world. Various strategies are used to extract information from remote sensing data. These strategies fall into two general categories called pixel based and object based. Most traditional pixel based approaches are based on the image pixels. By contrast, object based approaches are based on the objects which obtained by the segmentation of image data. Pixel based approaches only use digital number of pixels. Therefore, they face problems in complex urban areas. These problems cause from ignoring valuable information such as size, shape, and texture in high resolution images. Whereas object oriented approaches consider both spatial and spectral information. Accordingly, object oriented analysis produces better results especially in urban areas.

Image segmentation as the initial stage of object based analysis, has an important role in the performance of object oriented analysis. The more accurate segmentation is the better the results of classification will be.

Segmentation methods are usually categorized to two classes: edge-based and region-based techniques. Multi resolution segmentation, which was proposed by Baatz and Schäpe (2000), is one of the most powerful region based segmentation algorithms that have been implemented in commercial software on the market, eCognition (Definiens Imaging, 2009).

This algorithm is a bottom-up region merging process starting with one pixel and performs based on two heterogeneity criteria, geometrical and spectral. Smaller image objects are then merged into bigger ones which form segmentation with object on different scales.
Nevertheless, this algorithm needs a set of parameters which usually obtained by trial and error process that is tedious and time consuming (Tian and Chen, 2007). As a consequence, it is essential to find the optimum homogeneity parameters automatically.

There are many methods for the optimization of segmentation parameters such as neural network, ant colony, genetic algorithm, and so on. Among these algorithms, genetic algorithm is known as one of the powerful methods.

The genetic algorithm, which proposed by John Holland (1975), is based on evolution and natural process. To compare with other algorithms, genetic algorithm has many advantages in solving the optimization issues. At first, this algorithm produces initial population randomly and then evaluates the fitness each of chromosome in the population. Afterwards, the fittest chromosome is selected. At last, the next generation is produced by using crossover and mutation operators.

Genetic algorithm has been applied to many image processing applications such as image segmentation (Bhanu et al., 1995; Kim et al., 1998; Bhandarkar and Zhang, 1999; Tseng and Lai, 1999), image classification (Tseng et al., 2008; Wen et al., 2009; Stavrakoudis et al., 2011) and feature extraction (Krawiec and Bhanu, 2005; Puig and Garcia, 2006; Li et al., 2011) from remotely sensed images.

Chun and Yang (1996) used fuzzy and genetic algorithm to region based segmentation.

Van Coillie et al. (2007) selected the optimal features for object-oriented classification based on genetic algorithm.

\subsection{Related Works}

Up to now, many researches have been done in the field of multi resolution segmentation (Gamanya et al., 2007; Lucas et al., 2007; Mathieu et al., 2007; Mallinis et al., 2008; 
Schneevoigt et al., 2008; Dragut and Eisank, 2012). However, there have been few researches for determining optimal segmentation parameters.

Tian et al. (2007) presented a method for estimating parameters of multi resolution segmentation. His proposed framework was tested to segment features such as sports fields, roads, and residential buildings. It was found that best road segmentation results occurred in a small weight compactness and medium weight shape heterogeneity.

Dragut et al. (2010) proposed a method that was based on the local variance of object heterogeneity within a scene. Finally, appropriate scale was obtained by evaluating LV and changes of LV.

Lian et al. (2011) presented a method to determine scale factor based on standard deviation of the means and mean of standard deviations of image objects' brightness by using multi-resources images. It was proved relationship between spatial resolution and classification accuracy.

So this research aims to use genetic algorithm to determine optimal parameters by considering the road feature in IKONOS high resolution images.

This paper is outlined as follows. In section 2, a summary of multi resolution segmentation is presented and the proposed method is elaborated. The results are reported in section 3 . Finally, conclusions are described in section 4 .

\section{METHODOLOGY}

\subsection{Multi resolution Segmentation}

The multi resolution segmentation procedure could be summarized as the following (Baatz and Schäpe, 2000):

(1) Initially, each pixel in image is considered as a seed object. Then fusion factor $(f)$ is calculated for each of the neighbors of the selected seed object to find the best neighbor which has the minimum fusion factor.

(2) If best-fitting is not mutual, best candidate object is considered as new seed object and finds its best neighbor.

(3) When best-fitting is mutual, calculated fusion factor is compared to scale factor. If fusion factor be less than the square of scale factor, two objects will be merged.

(4) This process continues until no more merging is possible.

Fusion factor contains object features such as shape and color as observed in equation 1 .

$$
f=\mathrm{w}_{\text {shape }} \cdot \Delta \mathrm{h}_{\text {shape }}+\mathrm{w}_{\text {color }} \cdot \Delta \mathrm{h}_{\text {color }}
$$

$\Delta \mathrm{h}_{\text {color }}$ expresses difference in spectral heterogeneity that is defined as following:

$$
\Delta \mathrm{h}_{\text {color }}=\sum_{\mathrm{c}} \mathrm{w}_{\mathrm{c}}\left(\mathrm{n}_{\text {merge }} \cdot \sigma_{\mathrm{c}, \text { merge }}-\left(\mathrm{n}_{\mathrm{Obj} 1} \cdot \sigma_{\mathrm{c}}^{\mathrm{obj} 1}+\mathrm{n}_{\mathrm{Obj2} 2} \cdot \sigma_{\mathrm{c}}^{\mathrm{obj} 2}\right)\right)
$$

Where $\mathrm{n}_{\text {merge }}, \mathrm{n}_{\mathrm{obj} 1}, \mathrm{n}_{\mathrm{obj} 2}$ are number of pixels in merged object, object 1 and object 2 , respectively. The terms $\sigma_{\mathrm{c} \text {,merge }}, \sigma_{\mathrm{c}}^{\mathrm{Obj} 1}, \sigma_{\mathrm{c}}^{\mathrm{Obj} 2}$ represent standard deviations of merged object, object 1 and object 2 , respectively. $\mathrm{w}_{\mathrm{c}}$ is considered as weight of $\mathrm{c}$ spectral band.
The shape heterogeneity $\Delta \mathrm{h}_{\text {shape }}$ is calculated by compactness and smoothness heterogeneity of object.

$$
\Delta \mathrm{h}_{\text {shape }}=\mathrm{w}_{\text {comp }} \cdot \Delta \mathrm{h}_{\text {comp }}+\mathrm{w}_{\text {smooth }} \cdot \Delta \mathrm{h}_{\text {smooth }}
$$

Where

$$
\begin{aligned}
& \Delta \mathrm{h}_{\text {smooth }}=\mathrm{n}_{\text {merge }} \cdot \frac{l_{\text {merge }}}{\mathrm{b}_{\text {merge }}}-\left(\mathrm{n}_{\mathrm{Obj}-1} \cdot \frac{\mathrm{l}_{\text {Obj-1 }}}{\mathrm{b}_{\mathrm{Obj}-1}}+\mathrm{n}_{\mathrm{Obj}-2} \cdot \frac{\mathrm{l}_{\mathrm{Obj}-2}}{\mathrm{~b}_{\mathrm{Obj}-2}}\right) \\
& \Delta \mathrm{h}_{\text {comp }}=\mathrm{n}_{\text {merge }} \cdot \frac{\mathrm{l}_{\text {merge }}}{\sqrt{\mathrm{n}_{\text {merge }}}}-\left(\mathrm{n}_{\mathrm{Obj}-1} \cdot \frac{\mathrm{l}_{\text {Obj-1 }}}{\sqrt{\mathrm{n}_{\mathrm{Obj}-1}}}+\mathrm{n}_{\mathrm{Obj}-2} \cdot \frac{\mathrm{l}_{\mathrm{Obj}-2}}{\sqrt{\mathrm{n}_{\mathrm{Obj}-2}}}\right)
\end{aligned}
$$

As can be seen in equations (4) and (5), smoothness criterion equals the ratio factual border length $l$ and the perimeter $b$ of the bounding box. Meanwhile, compactness criterion equals the ratio factual border length 1 and the root of number of pixels in object.

Considering above equations, multi resolution segmentation needs six parameters including scale factor, weights of shape and color heterogeneity, weights of smoothness and compactness heterogeneity and weight of each spectral band. Among these parameters, weight of shape with color heterogeneity and weight of compactness with smoothness heterogeneity are complement. So the user should define four independent parameters namely scale factor, weights of compactness and shape heterogeneity, and weight of each spectral band. You can see a detailed description in (Baatz and Schape, 2000).

\subsection{The Proposed Method}

As mentioned above, four independent parameters are as input parameters of multi resolution segmentation. In this paper, we consider weight of each spectral band equal to 1 . So, we just optimize weights of shape and compactness heterogeneity and scale factor by using genetic algorithm. In other words, each chromosome in genetic algorithm has three genes that is coded in real values form. Weights of shape and compactness heterogeneity are set between 0.1 to 0.9 and 0 to 1 , respectively. Also, maximum scale factor is considered to 100.

In addition to crossover operation, the mutation operation is also essential for the GA because mutation causes anomalies in the reproductive cycle (Booker et al., 1997).

So in our approach, crossover probability is set to 0.8 , while the mutation probability equals 0.01 .

The fitness of chromosome has a vital role in genetic algorithm and controls the whole process. In our approach, fitness function which should be minimized is represented in following equation.

$$
\text { Fitness }=\left(\text { Area }_{\text {ref }}-\text { Area }_{\text {obt }}\right)+\mathrm{n}
$$

Where $\quad$ Area $_{\text {ref }}=$ Area of reference road segments Area $_{\text {obt }}=$ Area of generated road segments by GA $\mathrm{n}=$ number of generated road segments by GA

The method that we adopted is described below.

Firstly, initial population randomly is produced and their fitness is calculated. Afterwards, tournament selection method which selects parent with the highest fitness is chosen. After parent 
selection, a single point crossover is utilized to generate offspring. Finally, mutation operation is performed independently for each offspring.

In this way, next generation is produced and calculated their fitness. The process continues until a stopping criterion is satisfied.

Consequently, segmentation result evaluated by result obtained by using parameters found by the user.

In our case study, parameters should be selected by considering road feature.

Figure 1 shows the diagram of the implemented methodology in this research.

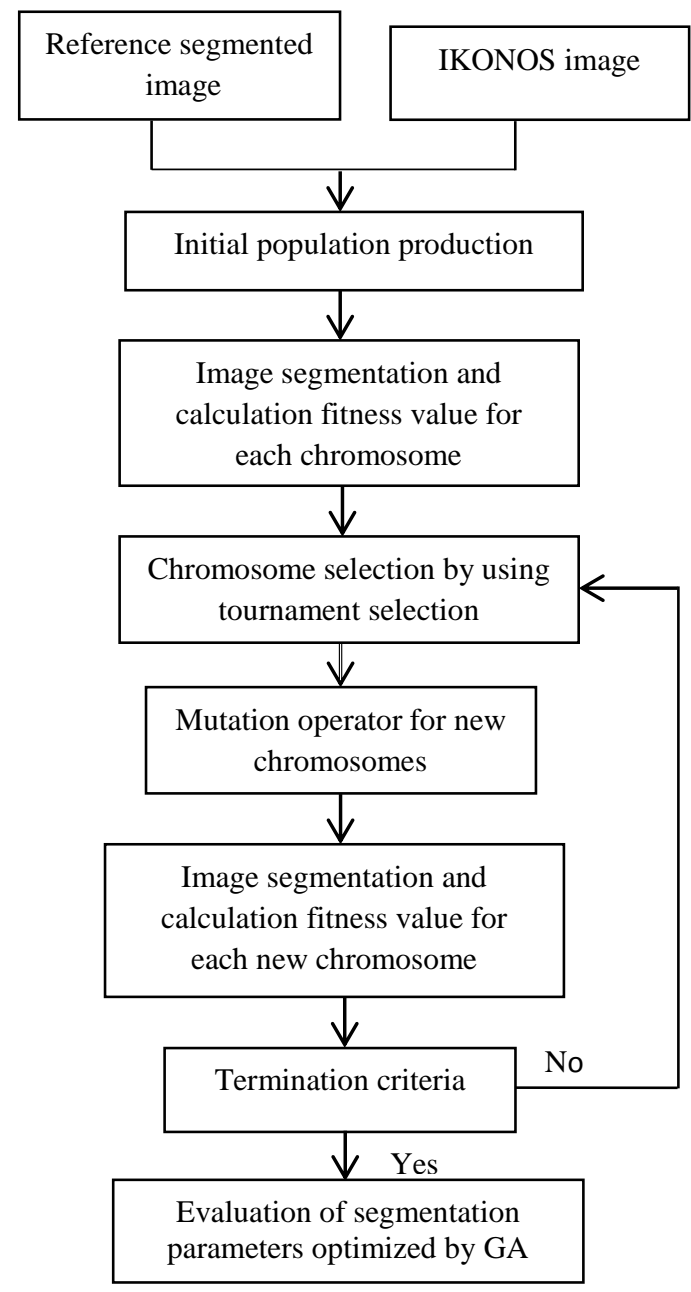

Figure 1. Flowchart of the proposed algorithm for optimizing parameters of multi resolution segmentation

\subsection{Study Area \& Input Data}

A pan-sharpened IKONOS satellite image with spatial resolution of $1 \mathrm{~m}$ is used in this paper. This image is taken from Shiraz city in Iran. Figure 2 shows the input image with their manually produced reference image.
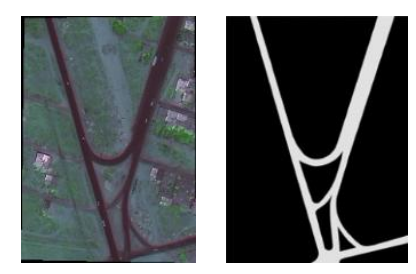

Figure 2. Pan-sharpened IKONOS image of Shiraz in Iran and its manually produced reference image

\section{RESULTS \& DISCUSSION}

In this paper, GA is used to address the problem of multi resolution segmentation parameters selection.

The manually optimized segmentation parameters have been found by the user.

Table 1 shows the obtained optimal parameters by GA and the user for input image. In order to verify the GA-optimized segmentation results, we generated error matrix. Overall accuracy and Kappa index derived from error matrix are listed in Tables 2.

\begin{tabular}{cccccc}
\hline \multicolumn{2}{c}{ Parameters found by GA } & \multicolumn{3}{c}{ Parameters found by the user } \\
\hline $\begin{array}{c}\text { Scale } \\
\text { factor }\end{array}$ & $\begin{array}{c}\text { Weight_ } \\
\text { Shape }\end{array}$ & $\begin{array}{c}\text { Weight } \\
\text { _ comp }\end{array}$ & $\begin{array}{c}\text { Scale } \\
\text { factor }\end{array}$ & $\begin{array}{c}\text { Weight_ } \\
\text { Shape }\end{array}$ & $\begin{array}{c}\text { Weight } \\
\text { comp }\end{array}$ \\
\hline 55.000 & 0.6000 & 0.2010 & 70 & 0.7 & 0.2 \\
\hline
\end{tabular}

Table 1. Segmentation parameters found by GA and the user

\begin{tabular}{ccc}
\hline & $\begin{array}{c}\text { GA-based } \\
\text { Segmentation }\end{array}$ & $\begin{array}{c}\text { User-based } \\
\text { Segmentation }\end{array}$ \\
\hline Accuracy & 96.4856 & 90.3536 \\
Kappa & 88.56 & 85.73 \\
\hline
\end{tabular}

Table 2.Overall accuracy and Kappa Index for GA-based and user-based segmentation

As noted in Table 2, segmentation accuracy and Kappa index by using parameters obtained by GA is higher than the user.

The result of segmentation based on GA-optimized and useroptimized parameters are shown in Figures 3. A cursory look at Figures 3 reveals that genetic algorithm gets better results. Visual assessment indicates the high capability of proposed method for choosing optimal parameters.

For closer investigation, some of road segments are shown by red circles in Figures 4 for GA-based and user-based segmentation. It is found from Figure that, mostly in the intersection areas, the road segmented by the user don't appear accurately. Whereas, GA could segments them well.

Another problem is related to some vehicles in road homogeneity surface. They perform as noises which make distribution in road segmentation and are considered as separated segments.

According to described above, it can be easily deduced that GA has significant effect on road segmentation results. 


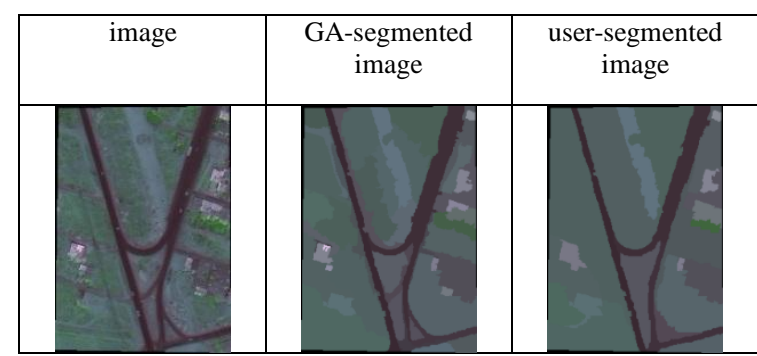

Figure3. The result of image segmentation by using parameters optimized by GA and the user

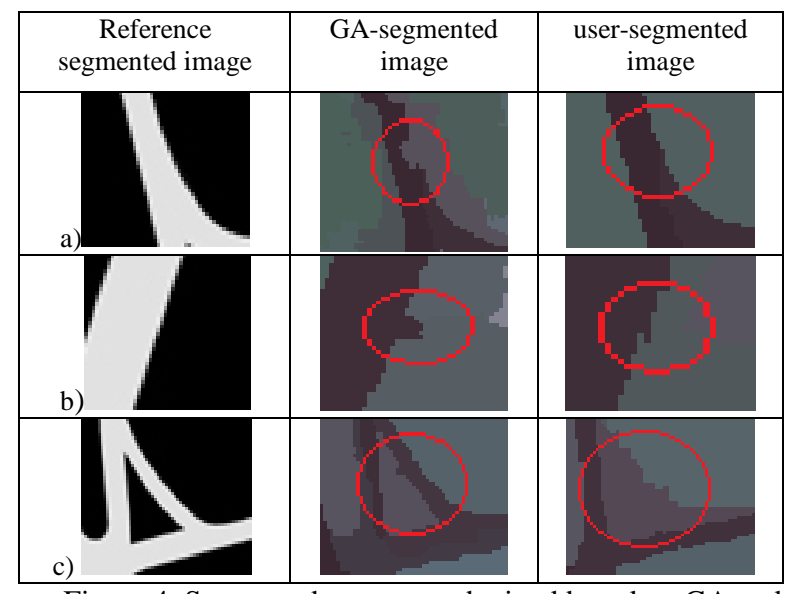

Figure 4. Some road segments obtained based on GA and the user

\section{CONCLUSION}

Although multi resolution segmentation has been utilized in recent years, choosing the suitable input parameters has remained a challenge. The selection of optimal parameters has been dependent to trial and error process which is tedious and time consuming. Thus, this paper presents optimization approach for parameters selection of multi resolution segmentation based on genetic algorithm.

The proposed method has been tested on IKONOS pansharpened image covering a part of Shiraz city in Iran. The cited work focused on road feature. So fitness function defined based upon roads in image. Finally, result evaluated by using assessment parameters derived from error matrix called overall accuracy and Kappa index.

The segmentation accuracy obtained by using GA-optimized parameters is higher than $96 \%$. As expected, the use of genetic algorithm yields a significant performance improvement in comparison with user-based. A visual assessment of results also confirms the importance of GA in finding parameters.

As seen from image, segmentation by using GA-optimized parameters could perform well in intersection areas in comparison with manual ones.

Something such as vehicles on road homogeneity surface cause spectral heterogeneity inside road object. So wrong selection of weight of spectral heterogeneity and consequently weight of shape heterogeneity can make roads don't appear linearly and normally. In addition, another problem can cause is to segment road and building as one object because of spectral similar of them. As a result, high weight of shape heterogeneity should be considered in complex urban areas. It should be noted that, very high weight of shape heterogeneity can cause some another problems.
The proposed method focusing on other interest objects might be worthy research in future. Additionally, fitness function designed for GA can be improved. More researches on a wider variety of scenes and using other type of sensors would be recommended as future works.

\section{References}

Baatz, M., Schape, A., 2000. Multi resolution segmentation-an optimization approach for high quality multi scale image segmentation. Angewandte Geographische Informations verarbeitung XII. Karlsruhe, Germany, pp. 12-23.

Bhandarkar, S. M., Zhang, H., 1999. Image Segmentation Using Evolutionary Computation. IEEE Trans. On Evolutionary Comp., Vol. 3, No. 1, pp. 1-21.

Bhanu, B., Lee, S., Ming, J., 1995. Adaptive Image Segmentation Using a Genetic Algorithm. IEEE Trans. On Systems, Man and Cybernetics, Vol. 25, No. 12, pp. 15431567.

Booker, L. B., Fogel, D. B., Whitley, D., Angeline, P. J., 1997. Recombination. In T. Bäck, D. B. Fogel, \& Z. Michalewicz (Eds.), Handbook of evolutionary computation (pp. C3.3:1-C3.3:27). Bristol and Oxford: IOP Publishing Ltd and Oxford University Press.

Chun, D. N., Yang, H. S., 1996. Robust Image Segmentation Using Genetic Algorithm with a Fuzzy Measure. Pattern Recognition, Vol. 29, No. 7, pp. 1195-1211

Definiens Imaging GmbH, eCognition 8.0 professional user guide, Munich, Germany.2009.

Dragut, L., Eisank, C., 2012. Automated object based classification of topography from SRTM data. Geomorphol. 141-142, 21-33.

Dragut, L., Tiede, D., R. Levick Sh., 2010. ESP: a tool to estimate scale parameter for multi resolution image segmentation of remotely sensed data. International Journal of Geographical Information Science. Vol. 24, No. 6, pp. 859871.

Gamanya, R., Maeyer, P. D., Dapper, M. D., 2007. An automated satellite image classification design using objectoriented segmentation algorithms: a move towards standardization. Expert Syst. Appl. 32(2), 616-624.

Holland, J. H., 1975. Adaptation in Natural and Artificial Systems, University of Michigan Press, Ann Arbor, MI, USA.

Kim, H. J., Kim, E. Y., Kim, J. W., Park, S. H., 1998. MRF Model Based Image Segmentation Using Hierarchical Distributed Genetic Algorithm. Electronics Letters, Vol. 34, No. 25, pp. 2394-2395.

Krawiec, K., Bhanu, B., 2005. Visual learning by coevolution feature synthesis. IEEE Trans. Syst. Man Cybern. 35(3), pp. 409-425.

Li, S., et al., 2011. An effective feature selection method for hyperspectral image classification based on genetic algorithm and support vector machine. Knowledge-Based Syst. 24(1), pp. $40-48$. 
Lian, L., Jianfei, C., 2011. Research on segmentation scale of multi-resources remote sensing data based on object-oriented. Procedia Earth and Planetary Science 2 .pp. 352 -357.

Lucas, R., et al., 2007. Rule-based classification of multitemporal satellite imagery for habitat and agricultural land cover mapping. ISPRS J. Photogramm. Remote Sens. 62(3), $165-185$.

Mallinis, G., et al., 2008. Object-based classification using Quickbird imagery for delineating forest vegetation polygons in a Mediterranean test site. ISPRS J. Photogramm. Remote Sens. 63(2), 237-250.

Mathieu, R., Freeman, C., Aryal, J., 2007. Mapping private gardens in urban areas using object-oriented techniques and very high-resolution satellite imagery. Landscape Urban Plann. 81(3), 179-192.

Puig, D., Garcia, M. A., 2006. Automatic texture feature selection for image pixel classification. Pattern Recognit. 39(11), pp. 1996-2009.

Schneevoigt, N. J., et al., 2008. Detecting alpine landforms from remotely sensed imagery: a pilot study in the Bavarian Alps. Geomorphol. 93(1-2), 104-119.

Stavrakoudis, D. G., Theocharis, J. B., Zalidis, G. C., 2011. A boosted genetic fuzzy classifier for land cover classification of remote sensing imagery. ISPRS J. Photogramm. Remote Sens. 66(4), pp. 529-544.

Tian, J., Chen, D. M., 2007. Optimization in multi-scale segmentation of high resolution satellite images for artificial feature recognition. Int. J. Remote Sens. 28(20), 4625-4644.

Tseng, M.H., et al., 2008. A genetic algorithm rule-based approach for land-cover classification. ISPRS J. Photogramm. Remote Sens. 63(2), pp. 202-212.

Tseng, D. C., Lai, C. C., 1999. A Genetic Algorithm for MRF Based Segmentation of Multi-Spectral Textured Images. Pattern Recognition Letters, Vol. 20, No. 14, pp. 1499-1510.

VanCoillie, F.M.B., Verbeke, L.P.C., De Wulf, Robert., 2007. Feature selection by genetic algorithms in object-based classification of IKONOS imagery for forest mapping in Flanders, Belgium. Remote Sens. Environ. 110(4), pp. 476-487.

Wen, X., et al., 2009. Multi scale modeling for classification of SAR imagery using hybrid EM algorithm and genetic algorithm. Prog. Nat. Sci. 19(8), pp. 1033-1036. 